\title{
VALIDAÇÃO DE METODOLOGIA PARA A DETERMINAÇÃO SIMULTÂNEA DOS ANTIOXIDANTES SINTÉTICOS EM ÓLEOS VEGETAIS, MARGARINAS E GORDURAS HIDROGENADAS POR CLAE/UV
}

\author{
Emy Takemoto* \\ Instituto Adolfo Lutz, Seção de Óleos, Gorduras e Condimentos, CP 1783, 01059-970 São Paulo - SP, Brasil \\ José Teixeira Filho
}

Faculdade de Engenharia Agrícola, Universidade Estadual de Campinas, Campinas - SP, Brasil

Helena Teixeira Godoy

Departamento de Ciências de Alimentos, Faculdade de Engenharia de Alimentos, Universidade Estadual de Campinas, Campinas - SP, Brasil

Recebido em 20/5/08; aceito em 13/1/09; publicado na web em 28/5/09

\begin{abstract}
VALIDATION OF METHODOLOGY FOR THE SIMULTANEOUS DETERMINATION OF SYNTHETIC ANTIOXIDANTS IN VEGETABLES OILS, MARGARINE AND VEGETABLES HYDROGENATED FATS BY HPLC/UV. The use of antioxidants either to prevent or retard food's lipids oxidation was approved after inquires that verified their security within a daily intake limit. In this study, the methodology was developed and validated for the analysis of synthetic antioxidants: propylgallate (PG), tertbutylhydroquinone (TBHQ), butylhydroxyanisole (BHA), octylgallate (OG) and butylhydroxytoluene (BHT) in vegetables oils, margarine and hydrogenated fats by high performance liquid chromatographic. The methodology revealed itself efficient, with recovery rates above $90 \%$ for all antioxidant substances, besides good linearity in concentration range of $40-240 \mathrm{mg} \mathrm{kg}^{-1}(\mathrm{r}=0,999)$, repeatability with $\mathrm{CV}<3,7 \%$ and limit of quantification $16.55,10.32,1.40,3.76$ and $9.30 \mathrm{mg} / \mathrm{kg}$ for BHT, BHA, PG, OG and TBHQ, respectively.
\end{abstract}

Keywords: synthetic antioxidants; HPLC; methodology.

\section{INTRODUÇÃO}

Um dos grandes problemas com os alimentos são as mudanças ou alterações que ocorrem durante o processamento, armazenamento e utilização. ${ }^{1}$

A maioria dos alimentos contém óleos e/ou gorduras, seja na forma pura ou como constituintes. Os óleos e gorduras têm uma grande importância pois, além de fornecerem energia, contribuem para o sabor dos alimentos (palatabilidade), são fontes de vitaminas lipossolúveis A, D, E e K, bem como a principal fonte de ácidos graxos essenciais. ${ }^{2}$

As alterações que óleos e gorduras sofrem são decorrentes de reações químicas que levam o alimento à deterioração. Essas reações podem ser a oxidação, reversão, hidrólise e polimerização. ${ }^{3}$ A reação de maior importância, tanto do ponto de vista econômico como nutricional, é a oxidação pois, devido à destruição de ácidos graxos essenciais e vitaminas lipossolúveis, ${ }^{4-6}$ acarreta alterações de odor, sabor, textura, consistência e aparência, assim como a perda de valor nutricional.

Os antioxidantes sintéticos são usados como aditivos alimentares para prevenir ou retardar a oxidação lipídica. São substâncias que tiveram seu uso aprovado em alimentos após investigações que comprovaram sua segurança dentro de um limite de ingestão diária; sendo assim, estão sujeitas a legislações específicas de cada país ou por normas internacionais. Desses antioxidantes sintéticos os mais utilizados pela indústria brasileira são: butil hidroxianisol (BHA), butil hidroxitolueno (BHT), galato de propila (PG) e terc-butil hidroquinona (TBHQ). No Brasil, ${ }^{7,8}$ as legislações vigentes permitem a adição, em óleos e gorduras de BHA e TBHQ no limite máximo de $200 \mathrm{mg} / \mathrm{kg}$; BHT, galatos de propila, dodecila e octila no limite de $100 \mathrm{mg} / \mathrm{kg}$ e, margarina (sobre o teor de gordura), no limite de $200 \mathrm{mg} / \mathrm{kg}$ de BHA, BHT, TBHQ e galato de propila.

*e-mail: etakemot@ial.sp.gov.br
Atualmente, muitos métodos analíticos têm sido pesquisados para detectar e quantificar essas substâncias. Esses métodos incluem desde a detecção qualitativa através de reações colorimétricas até métodos semiquantitativos e quantitativos, como espectrofotometria, voltametria, polarografia e métodos cromatográficos, tais como cromatografia em camada delgada - $\mathrm{CCD},{ }^{9,10}$ de permeação em gel - CPG, ${ }^{11}$ em fase gasosa - CG, ${ }^{12-15}$ líquida de alta eficiência CLAE ${ }^{16-22}$ e eletroforese capilar - CE. ${ }^{23}$ Dos métodos citados, CG e CLAE são os mais empregados para a separação e quantificação dos antioxidantes sintéticos.

Estudos de metodologias reportados na literatura descrevem diversos trabalhos para a determinação simultânea de antioxidantes sintéticos por CLAE empregando eluição isocrática e gradiente, diferentes características de colunas e fases móveis. A maioria dos trabalhos relatados tem utilizado eluição por gradiente ${ }^{18-22,24,25}$ embora eluição isocrática tenha sido usada em alguns casos. ${ }^{26-29}$

Métodos para a separação de antioxidantes em fase normal têm sido propostos por Van Niekerk e Du Plessis, ${ }^{30}$ Indik e Woollard, ${ }^{31}$ Anderson e Van Niekerk, ${ }^{26}$ mas são pouco empregados, já que a utilização de fase normal apresenta a desvantagem de necessitar de misturas complexas de solventes para a fase móvel, que são tóxicos tais como dioxano-hexano, ${ }^{30}$ hexano-cloreto de metileno-acetonitrila ${ }^{31}$ e hexano-1,4-dioxano-acetonitrila, ${ }^{22}$ enquanto que a fase reversa emprega solventes mais comuns como o metanol e acetonitrila e, portanto, tem a preferência dos pesquisadores. . $7-23,27,32-34^{-1}$

No Brasil, quase não existem dados sobre os níveis reais de antioxidantes presentes nos alimentos. Portanto, é de fundamental importância desenvolver um método analítico que seja adequado para a determinação simultânea dos antioxidantes sintéticos permitidos pela legislação brasileira. Recomenda-se que o método que seja simples, reprodutível e rápido, permitindo que órgãos oficiais fiscalizem e garantam que os alimentos estejam dentro de padrões de qualidade aceitáveis para consumo. 
Frente ao desenvolvimento tecnológico e a uma constante diversificação de produtos no mercado, é necessário o monitoramento desses antioxidantes nos alimentos. Além disso, a segurança desses compostos tem sido questionada nos últimos anos, devido ao seu potencial tóxico. ${ }^{35}$

O objetivo deste trabalho foi a validação de metodologia, utilizando cromatografia líquida de alta eficiência para determinação simultânea de antioxidantes sintéticos butil hidroxianisol (BHA), butil hidroxitolueno (BHT), galato de propila (PG), galato de octila (OG) e o tercbutil hidroquinona (TBHQ) em óleos vegetais, margarinas e gorduras hidrogenadas, priorizando a utilização de solventes menos tóxicos.

\section{PARTE EXPERIMENTAL}

\section{Materiais}

Foram utilizadas 2 amostras dos óleos de soja, canola, girassol e milho, 2 de margarina e 2 de gordura hidrogenada, sendo cada produto do mesmo fabricante, de mesma marca e diferentes lotes. Todas amostras foram adquiridas junto ao comércio da cidade de São Paulo/Brasil. Em algumas das amostras não havia no rótulo a declaração da presença de antioxidantes sintéticos. As determinações foram conduzidas em duplicata. Para a tomada de amostra foram utilizadas 2 embalagens, que tiveram o conteúdo homogeneizado antes da análise.

Os antioxidantes foram adquiridos das seguintes empresas: galato de propila (PG) da Sigma Chemical Co/USA, galato de octila (OG) da Aldrich Chemical Company Inc/USA, butil hidroxianisol (BHA), butil hidroxitolueno (BHT) e o terc-butil hidroquinona (TBHQ), com grau de pureza acima de 98\%, da Fluka Chemie/Suíça. Os solventes utilizados, todos grau CLAE, foram hexano e metanol (Omnisolv) da Merck/EM Science e acetonitrila (Chromar) da Mallinckrodt. O ácido acético PA foi obtido da Merck e filtrado em membrana Hv de $0,45 \mu \mathrm{m}$ de porosidade com $\varnothing=47 \mathrm{~mm}$, marca Sartorius. A água utilizada foi ultrapura, obtida no sistema Máxima Scientific da Elga (Inglaterra), e filtrada com membrana HA de $0,45 \mu \mathrm{m}$ de porosidade $\operatorname{com} \varnothing=47 \mathrm{~mm}$, marca Sartorius.

Uma solução estoque foi preparada para cada antioxidante (PG, TBHQ, BHA, OG e BHT). Pesou-se cerca de $50 \mathrm{mg}$ do antioxidante, transferiu-se para um balão volumétrico de $50 \mathrm{~mL}$ e completou-se o volume com metanol. A solução trabalho foi preparada a partir da diluição da solução estoque. Foi pipetado $1 \mathrm{~mL}$ da solução estoque para um balão volumétrico de $100 \mathrm{~mL}$, completado o volume com metanol, de maneira a obter uma concentração final de $0,01 \mathrm{mg} / \mathrm{mL}$. Todas as soluções foram filtradas em unidades filtrantes com membrana $\mathrm{Hv}$ de $0,45 \mu \mathrm{m}$, marca Millipore. As soluções padrões foram mantidas sob refrigeração a $4{ }^{\circ} \mathrm{C}$ e protegidas da luz por no máximo 2 dias para o TBHQ e 1 semana para os demais antioxidantes, segundo Page ${ }^{20}$ e Perrin e Meyer. ${ }^{22}$

\section{Método}

\section{Sistema CLAE-UV}

As análises cromatográficas foram realizadas empregando-se um cromatográfo líquido de alta eficiência, marca Shimadzu, composto de vários módulos: 2 bombas LC-10AD, detector de ultravioletavisível (SPD-10AV), um autoinjetor (SIL-10A), um degaseificador on-line (modelo DGU-14A-THE) e um software Class LC 10-A para aquisição de dados e controle total do sistema.

\section{Condições cromatográficas}

Foram empregadas duas colunas, uma Shim-pack ODS(M) de 5 $\mu \mathrm{m}, 250 \mathrm{~mm}$ x 4,6 i.d. e uma Chromolith de sílica, monolítica RP-18, $5 \mu \mathrm{m}, 100 \mathrm{~mm}$ x 4,6 mm i.d., para se avaliar a eficiência na separação dos antioxidantes sintéticos e a influência no tempo de análise.
A escolha na composição da fase móvel foi realizada de acordo com a melhor separação e resolução de cada antioxidante para sistemas de eluição isocrática e por gradiente. Como principais componentes da fase móvel estabeleceu-se metanol e água, que foram testados em diferentes proporções, com e sem a adição de ácido acético $5 \%$. A vazão em todos os testes foi mantida a $0,8 \mathrm{~mL} / \mathrm{min}$.

Os picos dos antioxidantes foram detectados através de detector de ultravioleta, operando a $280 \mathrm{~nm}$. A identificação foi feita por comparação de tempos de retenção de cada antioxidante com os tempos dos respectivos padrões analisados nas mesmas condições, bem como por cocromatografia.

\section{Preparo da amostra}

A extração dos antioxidantes foi feita com base no método da $\mathrm{AOAC}^{36}$ 983.15. Para a análise são tomadas 5,0 g de amostra de óleo de soja que são dissolvidas em $20 \mathrm{~mL}$ de hexano saturado com acetonitrila (saturar $300 \mathrm{~mL}$ de hexano em um funil de separação adicionando acetonitrila até que as 2 fases permaneçam em contato por 2 min de agitação). É feita a extração com três volumes de $50 \mathrm{~mL}$ de acetonitrila saturada com hexano (saturar $300 \mathrm{~mL}$ de acetonitrila em funil de separação adicionando hexano até que as 2 fases permaneçam em contato por 2 min de agitação). As alíquotas são reunidas e o solvente evaporado em evaporador rotativo, à temperatura de aproximadamente $40{ }^{\circ} \mathrm{C}$. O resíduo é ressuspendido em metanol e transferido para um balão volumétrico de $10 \mathrm{~mL}$, sendo o volume completado com metanol. Os extratos foram filtrados em membrana $\mathrm{Hv}$ com poros de $0,45 \mu \mathrm{m}$ antes de serem injetados no cromatógrafo.

A quantificação foi feita por padronização externa. Para a construção das curvas analíticas foram preparadas cinco soluções de trabalho diluídas com metanol, nas concentrações de 40, 80, 120, 160,200 e $240 \mathrm{mg} / \mathrm{kg}$ de cada antioxidante. Cada um dos seis pontos de cada curva representou a média de três determinações da mesma solução de trabalho. As curvas analíticas para cada antioxidante foram preparadas por regressão linear.

Validação do método

\section{$\underline{\text { Teste de recuperação }}$}

Os testes de recuperação foram realizados em óleo de soja, margarina e gordura hidrogenada, dissolvidos em $20 \mathrm{~mL}$ de hexano saturado com acetonitrila, onde foram adicionadas soluções padrões em dois níveis de concentração de 40 e $80 \mathrm{mg} / \mathrm{kg}$ de cada um dos antioxidantes (PG, TBHQ, BHA, OG e BHT), previamente dissolvidos em metanol e transferidos com auxílio de uma micropipeta. Procedeu-se, então, à extração dos antioxidantes como descrito na metodologia analítica.

\section{Repetitividade}

A avaliação da repetitividade foi realizada através de cinco determinações, em duplicata, em um nível de concentração de $30 \mathrm{mg}$ / $\mathrm{kg}$ de galato de propila (PG), terc-butil hidroquinona (TBHQ), butil hidroxianisol (BHA), galato de octila (OG) e butil hidroxitolueno (BHT) adicionados nas amostras de óleo de soja, margarina e gordura hidrogenada. A repetitividade foi calculada de acordo com Caulcutt e Boddy $^{37}$ (Equação 1).

$\mathrm{r}=t \sqrt{ } 2 \mathrm{sr}$

onde: $\mathrm{r}=$ repetitividade, com significância de 95\%; $t=t$ de Student e $\mathrm{sr}=$ estimativa do desvio padrão.

\section{Limites de detecção e quantificação}

Os limites de detecção e quantificação foram determinados utilizando-se equações de regressão linear, de acordo com Miller e Miller. ${ }^{38}$ 
Análise estatística

Foi realizada uma análise de regressão linear no nível de 95\% de confiança, para a obtenção de uma relação quantitativa entre as variáveis (Equação 2). Os intervalos de predição e confiança foram construídos junto ao modelo gerado, no mesmo nível de confiança. Toda a análise estatística foi obtida usando o pacote estatístico Minitab ${ }^{39}$ versão 13 (2000)/USA, fixando-se um nível de significância de 95\%.

$\mathbf{y}_{\mathbf{i}}=\beta_{\mathrm{o}}+\beta_{1} \mathrm{x}_{1}+\varepsilon_{\mathrm{i}}$

onde: $\mathbf{y}_{\mathbf{i}}=$ área do pico; $\beta_{\mathbf{o}}=$ coeficiente linear; $\beta_{1}=$ coeficiente angular; $\mathrm{x}_{1}=$ concentração do antioxidante sintético; $\varepsilon_{\mathrm{i}}=$ erro aleatório.

\section{RESULTADOS E DISCUSSÃO}

Para a otimização das condições para a separação simultânea dos antioxidantes sintéticos, inicialmente testou-se eluição isocrática. O tempo necessário, neste caso, para eluir os antioxidantes foi muito extenso, resultando em corridas muito longas, com picos largos de baixa resolução, levando os testes para a eluição por gradiente.

$\mathrm{O}$ fator de escolha da coluna Chromolith em relação à Shimpack é justificado pelas pressões muito elevadas, o que certamente diminuiria o tempo de vida útil da coluna e provocaria um desgaste maior do equipamento.

Foram testadas mais de dez combinações de metanol e água (acidificada ou não), com diferentes rampas de gradiente. A acidificação foi empregada em algumas fases móveis testadas em virtude dos dados apresentados por Ivanovic et al. ${ }^{40}$ que concluíram que além de manter os antioxidantes numa única forma (molecular ou ionizada) proporciona uma maior interação com a fase móvel, ocasionando a redução do tempo de análise. Para todas as fases móveis testadas, os principais problemas sempre se apresentavam na separação entre PG e TBHQ. O sistema de eluição que permitiu a melhor resolução dos cinco antioxidantes testados foi: iniciando com 55:45 (v/v) de metanol-água (acidificada com ácido acético 5\%, até $\mathrm{pH} 3,1$ ); chegando a 87:13 em $10 \mathrm{~min}$; permanecendo nessas condições por 5 min e retornando às condições iniciais após 20 min de corrida. A água acidificada evita a ionização dos compostos fenólicos ${ }^{41}$ que possuem pKa $>10 .{ }^{42}$ Consequentemente, na fase móvel os antioxidantes estarão presentes na forma não ionizada. A Figura 1a apresenta um cromatograma da separação da mistura de padrões.

Após a determinação das melhores condições cromatográficas, foi aplicada regressão linear para o estudo da linearidade e para avaliar numericamente a qualidade do ajuste do modelo foi realizada a análise da variância.
Os valores obtidos de $\mathrm{F}_{\mathrm{obs}}$. da análise da variância do ajuste dos modelos efetuados para PG, TBHQ, BHA, OG e BHT foram 75809,$54 ; 18467,00 ; 29267,45 ; 32739,85$ e 49073,93, respectivamente; em relação ao $\mathrm{F}_{\text {crit }}(4,38)$ sugerem que a regressão foi estatisticamente significativa.

Os resultados obtidos na regressão linear confirmaram a existência de uma relação linear entre as áreas e as concentrações préestabelecidas, com coeficiente de correlação (r) superior a 0,9993. Na Tabela 1 encontram-se as equações de regressão linear, coeficiente de correlação, $\mathrm{F}_{\mathrm{ob}}, p, \mathrm{R}^{2}$ ajustado, limites de detecção (LOD) e quantificação (LQ) do PG, TBHQ, BHA, OG e BHT.

A exatidão foi determinada através do estudo de recuperação dos antioxidantes, PG, TBHQ, BHA, OG e BHT, adicionados às amostras de óleo de soja, margarina e gordura hidrogenada em dois diferentes níveis de concentração, como mostra a Tabela 2. Na mesma tabela pode-se observar uma comparação com taxas de recuperações obtidas para alguns antioxidantes por outros pesquisadores. As taxas de recuperações obtidas neste trabalho são semelhantes às relatadas por Page ${ }^{19,20}$ em seus estudos colaborativos. Destacam-se as taxas de recuperação para o BHT, que foram superiores às da literatura. Como não foram encontrados níveis de recuperação para o galato de octila em óleo vegetal, apresentamos as taxas de recuperação obtidas por Page $^{20}$ para gordura hidrogenada, para efeito de comparação.

A Tabela 3 apresenta as faixas de repetitividade ${ }^{43}$ esperadas entre cinco determinações de cada antioxidante PG, TBHQ, BHA, OG e BHT em um nível de concentração, com 95\% de confiança. Desta forma espera-se que os valores obtidos por cinco determinações em duplicata, difiram dentro dos limites fornecidos pela repetitividade, com a confiança indicada. Os dados da Tabela 3 mostram que os valores obtidos foram repetitivos.

Os limites de detecção e quantificação (Tabela 1) foram determinados para os antioxidantes PG, TBHQ, BHA, OG e BHT utilizandose equações de regressão linear. ${ }^{38}$

Os limites de detecção encontrados neste trabalho foram 0,61 ; 2,36; 3.09; 1,13 e 4,96 mg/kg para PG, TBHQ, BHA, OG e BHT, respectivamente. Bannwart e Toledo ${ }^{44}$ relataram limites de detecção de 0,6; 2,7 e 3,0 mg/kg para BHA, BHT e TBHQ, respectivamente, utilizando um método de eluição isocrática.

A metodologia aqui validada foi aplicada a amostras de óleos vegetais, margarinas e gorduras hidrogenadas. Na Figura 1 são apresentados os cromatogramas referentes às amostras de óleo de soja, margarinas e gordura hidrogenada. Em nenhuma das amostras analisadas foi encontrado antioxidante não permitido pela legislação brasileira e em todos os casos os teores determinados estavam abaixo do limite máximo estabelecido (Tabela 4).

Tabela 1. Parâmetros da curva de regressão linear para faixa de concentração de 40 a $240 \mathrm{mg} / \mathrm{kg}$, no nível de confiança de $95 \%$, com limites de detecção (LOD) e quantificação (LQ) e $\mathrm{R}^{2}$ ajustado

\begin{tabular}{|c|c|c|c|c|c|c|}
\hline Antioxidante & Equação da reta & Fobs. & $P$ & $\mathrm{R}^{2}$ ajustado & LOD (mg/kg) & $\mathrm{LQ}(\mathrm{mg} / \mathrm{kg})$ \\
\hline PG & $\begin{aligned} y= & -14877,60+20259,92 x \\
& ( \pm 10617,55 \pm 73,58)\end{aligned}$ & 75809,54 & 0,00 & 99,97 & 0,61 & 1,40 \\
\hline TBHQ & $\begin{array}{c}y=6975,04+5849,77 x \\
( \pm 6208,30 \pm 43,05)\end{array}$ & 18467,00 & 0,00 & 99,89 & 2,36 & 9,30 \\
\hline BHA & $\begin{aligned} y= & 9163,62+5623,77 x \\
& ( \pm 4736,23 \pm 32,87)\end{aligned}$ & 29267,45 & 0,00 & 99,93 & 3,09 & 10,32 \\
\hline OG & $\begin{array}{c}y=-45641,3+15424,01 \\
( \pm 12293,95 \pm 85,24)\end{array}$ & 32739,85 & 0,00 & 99,94 & 1,13 & 3,76 \\
\hline BHT & $\begin{aligned} y= & 3386,43+3505,96 x \\
& ( \pm 2282,51 \pm 15,83)\end{aligned}$ & 49073,93 & 0,00 & 99,96 & 4,96 & 16,55 \\
\hline
\end{tabular}

$\mathrm{F}_{\text {crit }}(1,19 ; 0,05)=4,38$ 
Tabela 2. Recuperação dos antioxidantes adicionados em dois níveis de concentrações 40 e $80 \mathrm{mg} / \mathrm{kg}$ de cada um dos antioxidantes (PG, BHA, BHT, OG e TBHQ) no óleo de soja, margarina e gordura hidrogenada

\begin{tabular}{|c|c|c|c|c|c|c|c|c|c|c|c|c|c|c|c|}
\hline \multirow{4}{*}{ Antioxidante } & \multicolumn{15}{|c|}{ Autores } \\
\hline & \multirow{3}{*}{$\begin{array}{c}\text { Page }^{31} \\
\text { Recuperação } \\
\text { para } \\
\text { Óleos } \\
(\%)\end{array}$} & \multicolumn{2}{|c|}{ Page $^{32}$} & \multicolumn{12}{|c|}{ Neste trabalho } \\
\hline & & \multicolumn{2}{|c|}{$\begin{array}{l}\text { Recuperação } \\
\text { para }\end{array}$} & \multicolumn{4}{|c|}{$\begin{array}{l}\text { Recuperação } \\
\text { para Óleo* }\end{array}$} & \multicolumn{4}{|c|}{$\begin{array}{c}\text { Recuperação } \\
\text { para Margarina* }\end{array}$} & \multicolumn{4}{|c|}{$\begin{array}{c}\text { Recuperação } \\
\text { para Gordura Hidrogenada* }\end{array}$} \\
\hline & & $\begin{array}{c}\text { Óleos } \\
(\%)\end{array}$ & $\begin{array}{c}\text { Gordura } \\
\text { Hidrog. } \\
(\%)\end{array}$ & $\begin{array}{l}\text { Nível I } \\
(\mathrm{mg} / \mathrm{kg})\end{array}$ & $(\%)$ & $\begin{array}{l}\text { Nível II } \\
(\mathrm{mg} / \mathrm{kg})\end{array}$ & $(\%)$ & $\begin{array}{l}\text { Nível I } \\
(\mathrm{mg} / \mathrm{kg})\end{array}$ & $(\%)$ & $\begin{array}{l}\text { Nível II } \\
(\mathrm{mg} / \mathrm{kg})\end{array}$ & $(\%)$ & $\begin{array}{l}\text { Nível I } \\
(\mathrm{mg} / \mathrm{kg})\end{array}$ & $(\%)$ & $\begin{array}{l}\text { Nível II } \\
(\mathrm{mg} / \mathrm{kg})\end{array}$ & $(\%)$ \\
\hline \multirow[t]{3}{*}{ PG } & 87,7 & 90,9 & 97,0 & & & & & & & & & & & & \\
\hline & $\mathrm{a}$ & 95,2 & 102 & 40,22 & $101 \pm 3$ & 80,48 & $94 \pm 6$ & 44,22 & $98 \pm 4$ & 82,40 & $99 \pm 5$ & 43,72 & $96 \pm 3$ & 81,47 & $95 \pm 2$ \\
\hline & 97,3 & 96,9 & 104 & & & & & & & & & & & & \\
\hline \multirow[t]{3}{*}{ ТВHA } & 90,9 & 97,5 & 98,2 & & & & & & & & & & & & \\
\hline & $\mathrm{a}$ & 102 & 105 & 42,12 & $96 \pm 10$ & 80,32 & $90 \pm 8$ & 41,53 & $97 \pm 6$ & 81,92 & $97 \pm 4$ & 40,35 & $92 \pm 4$ & 80,18 & $94 \pm 2$ \\
\hline & 102,9 & 103 & 107 & & & & & & & & & & & & \\
\hline \multirow[t]{3}{*}{ BHA } & 97,3 & 98,0 & 95,2 & & & & & & & & & & & & \\
\hline & $\mathrm{a}$ & 99,1 & 96,5 & 40,68 & $101 \pm 3$ & 81,44 & $108 \pm 11$ & 40,18 & $100 \pm 9$ & 80,44 & $99 \pm 7$ & 42,18 & $96 \pm 7$ & 80,23 & $99 \pm 7$ \\
\hline & 101,2 & 101 & 101 & & & & & & & & & & & & \\
\hline \multirow[t]{3}{*}{ ВHT } & 81,9 & 83,1 & 77,4 & & & & & & & & & & & & \\
\hline & $\mathrm{a}$ & 83,8 & 79,4 & 40,32 & $97 \pm 4$ & 80,96 & $92 \pm 8$ & 42,51 & $98 \pm 6$ & 83,41 & $101 \pm 5$ & 40,30 & $96 \pm 5$ & 80,73 & $96 \pm 3$ \\
\hline & 86,4 & 85,1 & 80,2 & & & & & & & & & & & & \\
\hline \multirow[t]{3}{*}{ OG } & Não & Não & 93,5 & & & & & & & & & & & & \\
\hline & incluído & incluído & 96,2 & 40,54 & $100 \pm 5$ & 81,60 & $91 \pm 7$ & 40,67 & $95 \pm 5$ & 80,76 & $94 \pm 3$ & 42,08 & $92 \pm 3$ & 81,14 & $96 \pm 5$ \\
\hline & $\begin{array}{l}\text { neste } \\
\text { estudo }\end{array}$ & $\begin{array}{c}\text { neste } \\
\text { estudo }\end{array}$ & 96,8 & & & & & & & & & & & & \\
\hline
\end{tabular}

* Os resultados são médias de 5 determinações em duplicata.

Tabela 3. Repetitividade obtido através da adição de $30 \mathrm{mg} / \mathrm{kg}$ de PG, TBHQ, BHA, OG e BHT nas amostras de óleo de soja, margarina e gordura hidrogenada

\begin{tabular}{lcccccccccc}
\hline & \multicolumn{3}{c}{ Óleo de soja } & \multicolumn{3}{c}{ Margarina } & \multicolumn{3}{c}{ Gordura Hidrogenada } \\
Antioxidante & $\begin{array}{c}\mathrm{M} \pm \mathrm{DP} \\
(\mathrm{mg} / \mathrm{kg})\end{array}$ & $\begin{array}{c}\mathrm{CV} \\
(\%)\end{array}$ & $\begin{array}{c}\text { Repetitividade } \\
(\mathrm{r})\end{array}$ & $\begin{array}{c}\mathrm{M} \pm \mathrm{DP} \\
(\mathrm{mg} / \mathrm{kg})\end{array}$ & $\begin{array}{c}\mathrm{CV} \\
(\%)\end{array}$ & $\begin{array}{c}\text { Repetitividade } \\
(\mathrm{r})\end{array}$ & $\begin{array}{c}\text { M } \pm \text { DP } \\
(\mathrm{mg} / \mathrm{kg})\end{array}$ & $\begin{array}{c}\text { CV } \\
(\%)\end{array}$ & $\begin{array}{c}\text { Repetitividade } \\
(\mathrm{r})\end{array}$ \\
\hline PG & $31,25 \pm 0,36$ & 1,17 & 2,36 & $28,72 \pm 1,05$ & 3,70 & 2,31 & $26,75 \pm 0,83$ & 3,10 & 3,01 \\
TBHQ & $32,20 \pm 1,20$ & 3,72 & 4,31 & $33,45 \pm 0,82$ & 2,50 & 2,63 & $32,14 \pm 0,76$ & 2,40 & 2,67 \\
BHA & $29,05 \pm 0,58$ & 1,98 & 2,99 & $34,07 \pm 0,46$ & 1,40 & 3,11 & $31,28 \pm 1,02$ & 3,30 & 2,85 \\
OG & $35,37 \pm 0,45$ & 1,27 & 2,64 & $29,15 \pm 0,54$ & 1,90 & 2,64 & $33,15 \pm 1,13$ & 3,40 & 3,03 & 3,10 \\
BHT & $26,33 \pm 0,83$ & 3,15 & 3,58 & $27,11 \pm 0,63$ & 2,30 & 2,83 & $28,19 \pm 0,88$ & 3,86 \\
\hline
\end{tabular}

* n=5, média (M), desvio padrão (DP) e coeficiente de variação (CV).

Tabela 4. Resultados obtidos para análises de antioxidantes sintéticos em óleos vegetais, margarina e gordura hidrogenada

\begin{tabular}{|c|c|c|c|c|c|c|}
\hline \multirow{3}{*}{ Produto } & \multicolumn{6}{|c|}{ Concentração do Antioxidante (mg/kg) } \\
\hline & \multicolumn{2}{|c|}{ TBHQ } & \multicolumn{2}{|c|}{ BHA } & \multicolumn{2}{|l|}{ BHT } \\
\hline & $\mathrm{M} \pm \mathrm{DP}$ & $\mathrm{CV}(\%)$ & $\mathrm{M} \pm \mathrm{DP}$ & $\mathrm{CV}(\%)$ & $\mathrm{M} \pm \mathrm{DP}$ & $\mathrm{CV}(\%)$ \\
\hline Soja & $59,25 \pm 0,90$ & 1,52 & - & - & - & - \\
\hline Canola & $101,42 \pm 3,84$ & 3,79 & - & - & - & - \\
\hline Girassol & $\mathrm{nd}^{\mathrm{a}}$ & - & - & - & - & - \\
\hline Milho & $53,75 \pm 0,77$ & 1,43 & - & - & - & - \\
\hline Margarina & - & - & $\mathrm{nd}^{*}$ & - & $115,76 \pm 6,36$ & 5,59 \\
\hline $\begin{array}{l}\text { Gordura } \\
\text { Hidrogenada }\end{array}$ & - & - & $7,74 \pm 0,44$ & 5,69 & $58,34 \pm 0,75$ & 1,29 \\
\hline
\end{tabular}

* nd - não detectado; Resultados são representados como média (M), desvio padrão (DP) (n=4 para um lote) e coeficiente de variação (CV). 

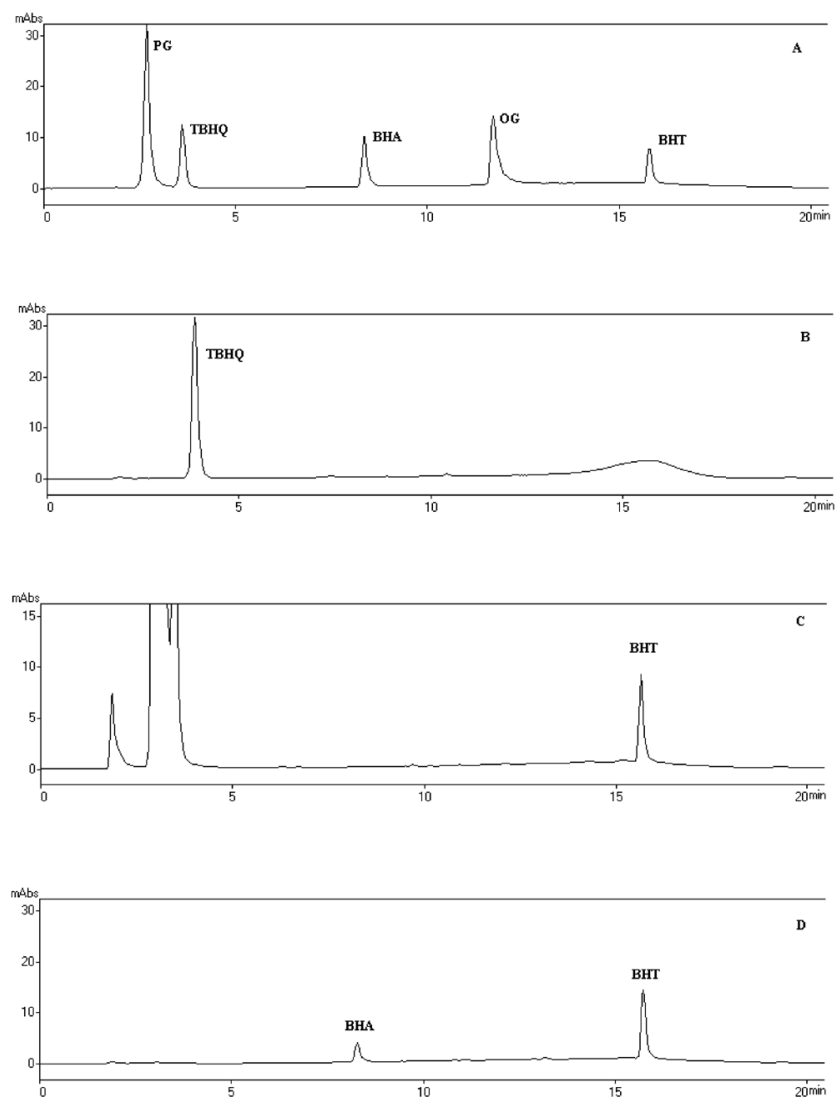

Figura 1. Cromatogramas da mistura padrão de antioxidantes sintéticos $P G, T B H Q, B H A, O G$ e BHT (20 mg/kg) (a), óleo de canola $(101 \mathrm{mg} / \mathrm{kg}$ de $T B H Q)(b)$, margarina (116 mg/kg de BHT) (c), gordura hidrogenada (8 $\mathrm{mg} / \mathrm{kg}$ de BHA e $58 \mathrm{mg} / \mathrm{kg}$ de BHT) (d), separados por CLAE. Condições cromatográficas: coluna Chromolit, $100 \mathrm{~mm} \times$ 4,6 mm d.i., $5 \mu \mathrm{m}$, vazão de $0,8 \mathrm{~mL} / \mathrm{min}$, fase móvel composta por metanol-água (acidificada com ácido acético 5\% até pH 3,1) iniciando com 55:45, chegando em 10 min a 87:13, permanecendo nessas condições por 5 min e retornando às condições iniciais após 20 min de corrida. Detecção em $280 \mathrm{~nm}$

Nos óleos vegetais de soja, canola e milho o único antioxidante encontrado foi TBHQ. Não foi encontrado nenhum antioxidante nas amostras de óleo de girassol. Já nas amostras de margarina foi detectada a presença de BHT e em gorduras hidrogenadas, a mistura de BHT e BHA.

Não foram detectados os antioxidantes PG, BHA, OG e BHT nos óleos de soja, girassol, canola e milho. Nas margarinas a indústria de alimentos tem utilizado o antioxidante BHT e nas gorduras hidrogenadas, a combinação do BHA e BHT.

\section{CONCLUSÕES}

A partir do estudo inicial dos parâmetros cromatográficos, realizou-se a otimização das condições para a determinação simultânea de cinco antioxidantes sintéticos - BHA, BHT, OG, PG e TBHQ permitidos pela legislação brasileira para uso em alimentos.

A acidificação da água, com redução do valor do $\mathrm{pH}$, foi fundamental para a obtenção de uma melhor resolução, fazendo com que a análise finalize em $20 \mathrm{~min}$.

A validação da metodologia mostrou-se bastante eficiente com taxas de recuperação superiores a $90 \%$ para os 5 antioxidantes sintéticos (PG, TBHQ, BHA, OG e BHT), boa linearidade e com limites de detecção semelhantes aos relatados na literatura.
O método através da utilização de solventes comuns e pelo tempo empregado na separação dos 5 antioxidantes em uma corrida cromatográfica de 20 min torna-o simples, rápido e reprodutivo, possibilitando que esta metodologia seja empregada em laboratórios de órgãos públicos e privados para a determinação simultânea dessas substâncias nos óleos, gorduras e margarinas comerciais.

\section{REFERÊNCIAS}

1. Shahidi, F.; Janitha, P. K.; Wanasundara, P. D.; Crit. Rev. Food Sci. Nutr. 1992, 32, 67.

2. Buck, D. F.; Food Additive User's Handbook, Blackie: London, 1991.

3. Adegoke, G. O.; Kuma, M. V.; Krishna, A. G. G.; Varadarj, M. C.; Sambaiah, K.; Lokesh, B. R.; J. Food Sci. Technol. 1998, 35, 283.

4. Byrd, S. J.; Cereal Foods World 2001, 46, 48.

5. Dziezak, J. D.; Food Technol. 1986, 40, 94.

6. Jadhavi, S. J.; Nimbalkar, S. S.; Kulkami, A. D.; Madhavi, D. L. Em Food Antioxidants: Technological, Toxicological, and Health Perspectives; Madhavi, D. L.; Deshpande, S. S.; Salunkhe, D. K.; Marcel Dekker: New York, 1995, $1^{\text {a }}$ ed.

7. Brasil. Ministério da Saúde, Conselho Nacional de Saúde; Resolução $n^{\circ}$ 04, de 24 de novembro de 1988; Aprovar a revisão das Tabelas I, III, IV e V referente a Aditivos Intencionais, bem como os Anexos I, II, III, IV e VII, todas do Decreto no 55.871, de 26 de março de 1965, Diário Oficial, Brasília, 19 de dezembro de 1988, Seção I, p. 24716-24723.

8. Brasil. Resolução RDC $n^{\circ} 23$, de 15 de fevereiro de 2005 Aprova "Regulamento Técnico que aprova o uso de aditivos alimentares, estabelecendo suas funções e seus limites máximos para a categoria de alimentos óleos e gorduras - subcategoria creme vegetal e margarinas", http://www.anvisa.gov.br/alimentos/legis/especifica/aditivos.htm, acessada em Outubro 2008.

9. Sahasrabudhe, M. R.; J. AOAC 1964, 47, 888.

10. van Peteghem, C. H.; Dekeyser, D. A.; J. AOAC 1981, 64, 1331.

11. Doeden, W. G.; Bowers, R. H.; Ingala, A. C.; J. Am. Oil Chem. Soc. 1979, 56, 12.

12. Choong, Y. M.; Lin, H. J.; J. Food Drug Anal. 2001, 9, 20.

13. González, M.; Gallego, M.; Valcárcel, M.; J. Chromatogr., A 1999, 848, 529.

14. Hartman, K. T.; Rose, L. C.; J. Am. Oil Chem. Soc. 1970, 47, 7.

15. Kline, D. A.; Joe, F. L.; Fazio, T.; J. AOAC 1978, 61, 513.

16. Karovicová, J.; Simko, P., J. Chromatogr., A 2000, 882, 271.

17. Oishi, M.; Matsuda, T.; Nojiri, S.; Saito, K.; J. Food Hyg. Soc. Japan 2002, 43, 104 .

18. Page, D. P.; J. AOAC 1979, 62, 1239.

19. Page, D. P.; J. AOAC 1983, 66, 727.

20. Page, D. P.; J. AOAC 1993, 76, 765.

21. Page, D. P.; Charbonneau, C. F.; J. AOAC 1989, 72, 259.

22. Perrin, C.; Meyer, L.; Food Chem. 2002, 77, 93.

23. Boyce, M. C.; Electrophoresis 2001, 22, 1447.

24. Andrikopoulos, N. K.; Brueschwellwer, H.; Felber, H.; Taeschler, Ch.; J. Am. Oil Chem. Soc. 1991, 68, 359.

25. Pinho, O.; Ferreira, I. M. P. L.; Oliveira, M. B. P. P.; Ferreira, M. A.; Food Chem. 2000, 68, 353.

26. Anderson, J.; van Niekerk, P. J.; J. Chromatogr. 1987, 394, 400.

27. Constante, E. G.; Grasas y Aceites 1975, 26, 150.

28. Hall III, C. A.; Zhu, A.; Zeece, M. G.; J. Agric. Food Chem. 1994, 42, 919.

29. Rustan, I.; Damiano, M. A.; Lesgards, G.; Annales des Falsifications, de I'Expertise-Chimique et Toxicologique 1993, 86, 201.

30. van Niekerk, P. J.; Du Plessis, L. M.; J. Chromatogr. 1980, 187, 436.

31. Indyk, H.; Woollard, D. C.; J. Chromatogr. 1986, 356, 401.

32. Chen, B. H.; Fu, S. C.; Chromatographia 1995, 41, 43. 
33. Yankah, V. V.; Ushio, H.; Ohshima, T. E.; Koizumi, C.; Lipids 1998, 33, 1139.

34. Sin, D. W. M.; Wong, Y. C.; Mak, C. Y.; Sze, S. T.; Yao, W. Y.; J. Food Composition and Analysis 2006, 19, 784.

35. Madhavi, D. L.; Deshpande, S. S.; Salunkhe, D. K. Em ref. 6.

36. A.O.A.C.; Official Methods of Analysis of AOAC International, $17^{\text {th }} \mathrm{ed}$., Association of Official Analytical Chemists: Arlington, 2003.

37. Caulcutt, R.; Boddy, R.; Statistic for Analytical Chemists, $1^{\text {st }}$ ed., Chapman and Hall: New York, 1983.

38. Miller, J. C.; Miller, J. N.; Statistic for Analytical Chemistry, $3^{\text {rd }}$ ed., Ellis Horwood and Prentice Hall: New York, 1993.
39. MINITAB for windows, [Minitab-Inc, USA] Versão. 13.31. 2000. CDroom.

40. Ivanovic, D.; Medenica, M.; Nivaud-Guernet, E.; Guernet, M.; Chromatographia 1995, 40, 652.

41. López, M.; Martinez, F.; Del Valle, C.; Orte, C., Miró, M.; J. Chromatogr. A 2001, 922, 359

42. Shiu, W-Y.; Ma, K-C.; Varhannickova, D.; Mackay, D.; Chemosphere 1994, 29, 1155

43. INMETRO; DOQ-CGCRE-008. Revisão: 01, 2003.

44. Bannwart, X. Z.; Dissertação de Mestrado, Universidade Estadual de Campinas, Brasil, 2000. 